\title{
A propósito de los sistemas electorales*
}

\section{Introducción}

En la actualidad, el debate sobre los sistemas electorales ocupa un lugar importante en la literatura política y sociológica. Desde que, en la década de los ochenta, se comenzó a discutir en América Latina el problema de la transición a la democracia, se volvió a revitalizar el tema de las elecciones como mecanismo privilegiado para elegir y revocar gobernantes.

Antes del advenimiento de las dictaduras militares conosureñas de los años sesenta y setenta, países como Chile, Argentina y Uruguay habían consolidado sistemas electorales fuertes y con un grado notable de legitimidad ante la sociedad civil. Las dictaduras militares socavaron esa legitimidad y dieron al traste con el tipo de democracia política que dichos sistemas electorales garantizaban.

Cuando se inician las transiciones a la democracia en América del Sur, en la década de los ochenta, el primer desafío de los actores involucrados en ellas es el de reconstituir los mecanismos democráticos violentados por los militares. Indudablemente, uno de los temas que ocupó un lugar privilegiado en la agenda de discusión fue el de los sistemas electorales como mecanismo fundamental para asegurar la elección periódica, transparente y competitiva de nuevos gobernantes.

Por otra parte, no se trataba sólo de reestablecer los sistemas electorales previos a la llegada de los militares al poder, sino también de pro- ceder a su reestructuración, corrigiendo sus vicios más notorios y adecuándolos a los nuevos tiempos. A esta necesidad es a la que respondió toda la literatura sociológica y política mencionada antes.

Como resultado de esas reflexiones teóricas y políticas ahora existe un amplio consenso, tanto en América del Sur como en Centroamérica, de que la democracia (política) exige no sólo la realización de elecciones periódicas, transparentes, pluralistas y competitivas, sino también que el voto de cada miembro de la sociedad sea en verdad significativo. Y que esto sea así, corre por cuenta de los sistemas electorales.

A continuación vamos a tratar algunos aspectos teóricos que son básicos para el estudio de los sistemas electorales.

\section{Elementos teóricos}

Pues bien, un sistema electoral puede ser definido como el conjunto de procedimientos, reglas y mecanismos de acuerdo con los cuales los electores expresan su voluntad a través del voto. Los votos se traducen en escaños o bancadas parlamentarias. Dicho de otro modo, cuando se discuten los sistemas electorales, el énfasis recae sobre las reglas mediante las que se asignan las bancas en el parlamento o la asamblea; y las mismas o bien pueden ser mayoritarias o proporcionales, o bien pueden ser una combinación de ambas. Otros aspectos que suelen debatirse tienen relación con la división del país en circunscripciones, las for-

*Texto reelaborado de una charla ofrecida por el autor en el Colegio de Altos Estudios Estratégicos (CAEE), el 24 de septiembre de 1997. 
mas de candidatura y los procedimientos de votación. Estos elementos, para tener vigencia plena, deben ser contemplados en la legislación que rige a la sociedad de que se trate, pues atañen a su propia definición como régimen político.

Según la literatura sociológica y política, existen dos tipos básicos de sistemas electorales. El primero es conocido como el "mayoritario-pluralista", en donde quien gana es el que tiene más votos; y el segundo es el "proporcional", que pretende que todas las opiniones estén representadas en el parlamento o, como dice Sartori, el que tiene como propósito que el parlemento sea un "espejo del electorado". Por lo demás, ambos tipos de sistema electoral pueden ser caracterizados de una forma más sutil: preguntándose cómo se asignan los escaños y qué es lo que se busca en cada uno.

Dicho brevemente, en el mayoritario-pluralista los escaños se asignan según la mayoría de votos y su fin es lograr una mayoría parlamentaria; mientras que en el proporcional, los escaños se asignan según la proporción de votos obtenidos y lo que se busca es que el parlamento refleje lo más fielmente posible los intereses del electorado. Con todo, en la práctica, ambos tipos de sistema electoral pueden mezclarse, como es el caso de aquellos parlamentos mayoritarios conseguidos por una asignación proporcional de diputados o aquellos parlamentos-espejo del electorado obtenidos por una asignación mayoritaria. También es relativamente normal la convivencia de ambos sistemas electorales cuando se utiliza, por ejemplo, la regla mayoritaria para la elección del Presidente de la República y los Concejos Municipales, y la regla de la proporcionalidad para la elección de diputados.

Pero, en definitiva, ¿qué es lo que se busca cuando se implementa uno u otro tipo de sistema electoral? Esta es una pregunta por los objetivos políticos de los sistemas electorales, ya que detrás de la dimensión técnica de los mismos están los intereses de los grupos de poder que pretenden servirse -y se sirven de hecho- de aquéllos.

Comencemos por el sistema mayoritario. Como es ampliamente aceptado en la actualidad, con éste se pretende la eficacia gubernamental; es decir, se busca asegurar al partido que va a gobernar, o a una coalición de partidos, la mayoría sólida en el parlamento o la asamblea legislativa. Esto se garantiza una vez que se haya obtenido la ma- yoría - una mayoría simple puede bastar-, el partido o la coalición ganadores acceden a toda la cuota de poder en disputa. Pueden implementar políticas y tomar decisiones sin mayores impedimentos $y$, en consecuencia, realizar una gestión política -esa es la apuesta - más eficaz. Detrás de este razonamiento está la idea de que el debate político - sobre todo el alimentado por las minorías- obstaculiza la gobernabilidad, por lo que es mejor eliminar la posibilidad de que esas minorías no sólo accedan al gobierno, sino que puedan entorpecer la labor de quienes sí están en capacidad de gobernar.

Los defensores del sistema proporcional, por su parte, quieren un parlamento que refleje la opinión de los ciudadanos y de las fuerzas sociales y políticas. Buscan que la proporción de escaños que se asigna a un partido sea similar a la proporción que representan sus votos en el total. Tienen que resolver dos problemas: cuántas bancas corresponden a cada partido y quién decide cuáles personas las ocuparán. Esto da pie a interminables cálculos políticos, los cuales nunca dejan satisfechos plenamente a los interlocutores, ya que cada uno cree tener la mejor propuesta acerca de cuál es la persona más idónea y de cuál es el número de bancas que deben ser asignadas a cada institutución política. Para quienes apuestan por la proporcionalidad, el problema no es asegurar la gobernabilidad, sino crear un sistema político más equitativo; es decir, un sistema político en el que cada partido tenga la cuota de poder que le corresponde según su proporción de votos. Esto último obliga, en este sistema, a formular cálculos de diversa naturaleza para establecer la proporcionalidad de los resultados electorales.

¿Eficacia política o equidad política? En torno a esta disyuntiva se organizan los sistemas electorales $\mathrm{y}$, aunque aparentemente la elección por uno es obligada -y excluyente del otro-, ni teórica ni empíricamente puede justificarse exclusión alguna, pues lo más realista es la búsqueda de soluciones intermedias que permitan, hasta donde sea posible, tanto la eficacia gubernamental como la equidad en la distribución del poder.

Por lo demás, una vez que se ha establecido un sistema electoral y han asumido los objetivos políticos que se pretenden alcanzar, no se puede pasar desapercibido otro aspecto importante: que los sistemas electorales no sólo responden a objetivos políticos - tal como se cristaliza en los partidos- 
sino que tienen un fuerte impacto en el sistema partidario al que también ayudan a moldear.

En los años 1950 y 1951, Maurice Duverger formúlo tres leyes sociológicas acerca del impacto de los sistemas electorales sobre el sistema de partidos: (a) la representación proporcional que tiende a la formación de muchos partidos independientes; (b) el escrutinio mayoritario en dos vueltas que tiende a la formación de muchos partidos aliados entre sí, y (c) el escrutinio mayoritario en una sola vuelta que tiende a producir un sistema bipartidista.

Estas "leyes", pese a haber sido fuertemente criticadas cuando fueron formuladas, en la actualidad han sido recuperadas por autores como Giovanni Sartori, quien las ha convertido en leyes tendenciales. Por otra parte, los teóricos aceptan, también tendencialmente, que a mayor fragmentación social es más probable el surgimiento de un sistema electoral proporcional y el surgimiento de un sistema de partidos pluripartidista. E, inversamente, a mayor homogeneidad social será más probable que se opte por el sistema de mayoría relativa, pero también es probable que surja un sistema bipartidista o un pluripartidismo limitado (Ver Rolando Franco. "Sistemas electorales y gobemabilidad", Transiciones a la democracia en Europa y América Latina, México, 1991, pp. 171-194).

Dicho lo anterior, ¿cuál es el sistema electoral dominante en América Latina? Pues bien, en América Latina, el sistema electoral predominante es el de representación proporcional. Además, hay un consenso bastante generalizado acerca de lo difícil que sería, pese a los retos que plantea la gobemabilidad democrática, convertirlo en un sistema mayoritario. Por eso, los esfuerzos que se han hecho hasta ahora, recogidos en las reformas electorales, se han encaminado a la introducción de modificaciones importantes al sistema proporcional, sin intentar una transformación radical del mismo. Esas modificaciones han apuntado en tres direcciones: el diseño de las circunscripciones; los umbrales de representación, y la fórmula de la asignación de bancas.

(a) El diseño de las circunscripciones -es decir, cuántos diputados tiene cada región y cuántos votos son necesa- rios para elegirlos - es de particular importancia por la posibilidad de éxito de los partidos pues, en muchos casos - como en El Salvador-, cada voto no es igual a los demás. Idealmente, se debería usar un procedimiento uniforme de asignación de escaños en todo el territorio, fijando como criterio el porcentaje de población, la cantidad de votantes o los votos válidos que se emitan en cada una. Pero, en la práctica, existen diversas razones que llevan a apartarse de este ideal, como es el caso de aquellos Estados federados en los que cada provincia es representada en la Cámara Alta por uno o más senadores, independientemente de su población. También sucede que los resultados electorales suelen ser manipulados por una delimimitación de las circunscripciones, que garantiza que determinado partido pueda llevarse la mayor cantidad de escaños.

Así pues, según sea el tamaño de las circunscripciones (es decir, la cantidad de parlamentarios que se eligen en ella y los votos que se requieren para la elección de cada uno) así variará la proporcionalidad y será diferente el umbral que se establece para que los pequeños partidos puedan ingresar en el parlamento.

(b) Los umbrales de representación hacen referencia a los mínimos que necesita cada partido para tener representación parlamentaria. Los distintos estados sitúan esos umbrales en diversos niveles, pero el propósito común es evitar la presencia de partidos extremadamente pequeños que dificulten la funcionalidad del parlamento. Cada Estado, pues, establece qué porcentaje de votos es necesario para obtener representación parlamentaria e, incluso, qué porcentaje de votos es necesario para que los partidos continúen vigentes. El pro-

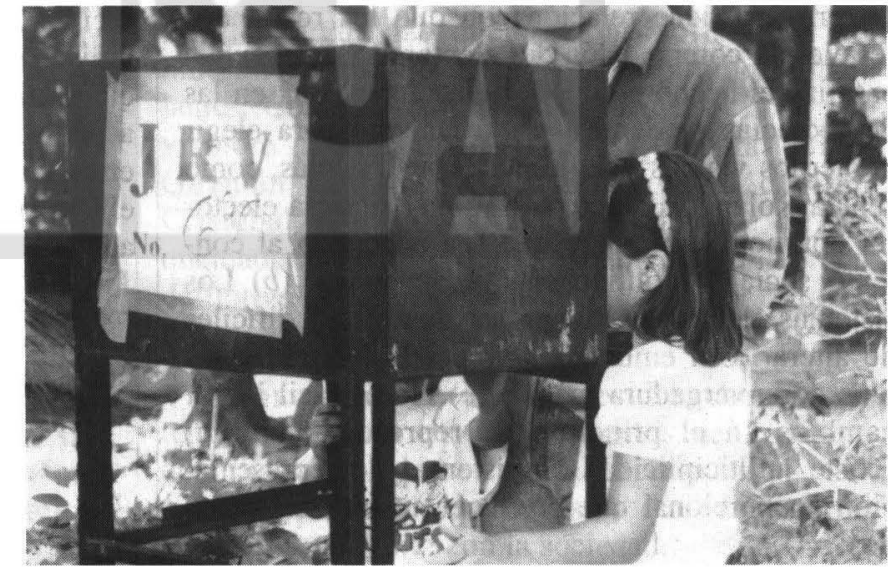


pósito es limitar el acceso de los partidos más pequeños al parlamento, lo cual garantizará a los partidos ganadores lograr los consensos necesarios para gobernar. De este modo, en aras de la eficacia, se pone un límite a la equidad, sin que ésta última deje de tener importancia.

(c) La fórmula de asignación de bancas. MienIras que el sistema mayoritario no plantea mayores problemas respecto de quién gana y cuánto, el sistema de representación proporcional exige establecer algún mecanismo para determinar la relación que debe existir entre los votos y las bancas. Estos mecanismos básicamente son dos: (1) determinar cuántos votos son necesarios para conseguir una banca; para lo cual, si una lista ha alcanzado esa cifra obtiene automáticamente un escaño; (2) dividir la cantidad de votos obtenidos por cada partido en una serie continua de números, de modo que emerjan series numéricas decrecientes para cada uno. Las bancas se van asignando al cociente más alto.

La implementación de ambos mecanismos no es fácil ni transparente, pues - para acercarse al ideal de proporcionalidad- requieren no sólo que la población en edad de votar esté debidamente registrada en el padrón electoral -y que efectivamente vote-, sino que el número de votos con el que se elige a un diputado sea el más idóneo para la circuscripción de que se trate, y que el número de diputados asignados a la misma sea acorde con su población electoral.

\section{Conclusión}

Para resumir podemos decir lo siguiente. En la implementación de un sistema electoral se ponen en juego dos cosas: la equidad y el pragmatismo. La primera tiene relación con que los resultados reflejen la composición del electorado; la segunda enfatiza el hecho de que las elecciones son, en las democracias modernas, el mecanismo para elegir gobiernos viables y legítimos. Por lo demás, como señala Rolando Franco: $(a)$ no hay sistema electoral óptimo. Se requiere que sea adecuado al contexto social y político en que se aplica. (b) Los sistemas electorales suelen ser durables y difíciles de alterar. Sin embargo, hay posibilidad de cambios de envergadura menor. (c) No es fácil lograr cambios en el principio de representación. (d) Existe multiciplicidad de sistemas de representación proporcional que producen resultados variados.
La democracia, como ha dicho Umberto Cerroni, es un régimen político que permite contar las cabezas sin romperlas. Ese conteo de cabezas debe asegurar - ese es el propósito esencial de los sistemas electorales- que cada una se traduzca en un voto y que éste tenga un valor igual para todos los ciudadanos. En países como El Salvador, todavía unos ciudadanos valen más políticamente que otros - los que votan versus los que no votan-y el voto de algunos vale más que el de otros - los que eligen diputados con menos votos en un departamento respecto a los que eligen igual número de diputados con más votos. Pero ello no debe ser un motivo para desconfiar o renegar de la democracia y sus valores, sino que más bien debe alentar aquellas prácticas y actitudes orientadas a fortalecerla. En El Salvador son cada vez más urgentes las reformas electorales que propicien una equidad política más acorde con los diversos grupos de interés, cuyas demandas no tienen eco político alguno. Ahora mismo se discute mucho acerca de la necesidad de introducir mejoras en el sistema electoral. Por supuesto, prácticamente nadie sugiere que de un sistema proporcional se pase a uno mayoritario, no sólo por lo difícil que ello sería, sino por que aquél también tiene sus desventajas. Existe, pues, un cierto consenso acerca de reformar algunos aspectos del sistema proporcional vigente.

En primer lugar, una reforma importante tiene relación con el establecimiento claro de los criterios para asignar tal o cual número de diputados a cada departamento. En segundo lugar, se ha discutido la necesidad de hacer que cada ciudadano pueda traducirse en un voto. Para esto es básico crear las condiciones para que cada uno de los habitantes de El Salvador pueda votar con la mayor facilidad. En tercer lugar, se ha planteado como un grave problema el que un $\mathbf{4 8}$ por ciento de los ciudadanos elija a los 84 diputados. Habría que aspirar a que un 100 por ciento de los ciudadanos en edad de votar puedan asistir a las umas, pero ello supone tener un sistema de empadronamiento adecuado.

Si se avanza en estas reformas, seguramente no tendremos el mejor sistema electoral del mundo, pero sí uno mejor que el actual. 